\title{
Insufficient evidence to assess the effectiveness of antibiotics for irreversible pulpitis
}

\section{Abstracted from \\ Fedorowicz Z, van Zuuren EJ, Farman AG, Agnihotry A, AI-Langawi JH.}

Antibiotic use for irreversible pulpitis. Cochrane Database Syst Rev 2013; 12: Art. No: CD004969. DOI: 10.1002/14651858.CD004969.pub3.

Address for correspondence: Luisa Fernandez Mauleffinch, Review Group Co-ordinator, Cochrane Oral Health Group, MANDEC, School of Dentistry, University of Manchester, Higher Cambridge Street, Manchester M15 6FH, UK. E-mail: luisa.fernandez@manchester.ac.uk

\section{Question: Are systemic antibiotics effective in the management of acute pulpitis?}

Data sources The Cochrane Oral Health Group's Trials Register, the Cochrane Central Register of Controlled Trials (CENTRAL), Medline, Embase and the US National Institutes of Health Trials Register. Study selection Randomised controlled trials (RCTs) which compared pain relief with systemic antibiotics and analgesics, against placebo and analgesics in the acute preoperative phase of irreversible pulpitis. Data extraction and synthesis Study selection, risk of bias assessment and data extraction were carried out independently by two reviewers. Pooling of data was not possible and a descriptive summary is presented. Results One trial assessed at low risk of bias, involving 40 participants, was included in this update of the review. The quality of the body of evidence was rated low for the different outcomes. There was a close parallel distribution of the pain ratings in both the intervention and placebo groups over the seven-day study period.

There was insufficient evidence to claim or refute a benefit for penicillin for pain intensity. There was no significant difference in the mean total number of ibuprofen tablets taken over the study period: 9.2 (standard deviation (SD) 6.02) in the penicillin group versus 9.6 (SD 6.34 ) in the placebo group; mean difference -0.40 ( $95 \%$ confidence interval $(\mathrm{Cl})-4.23$ to $3.43 ; \mathrm{P}$ value $=0.84)$. This applied equally for the mean total number of Tylenol tablets: 6.9 (SD 6.87) used in the penicillin group versus 4.45 (SD 4.82) in the placebo group; mean difference $2.45(95 \% \mathrm{Cl}-1.23$ to 6.13 ; P value $=0.19)$. Our secondary outcome on reporting of adverse events was not addressed in this study. Conclusions This systematic, review which was based on one lowpowered small sample trial assessed as a low risk of bias, illustrates that there is insufficient evidence to determine whether antibiotics reduce pain or not compared to not having antibiotics. The results of this review confirm the necessity for further larger sample and methodologically sound trials that can provide additional evidence as to whether antibiotics, prescribed in the preoperative phase, can affect treatment outcomes for irreversible pulpitis.

This paper is based on a Cochrane Review published in the Cochrane Library 2013, issue 12 (see www.thecochranelibrary.com for information). Cochrane Reviews are regularly updated as new evidence emerges and in response to feedback, and the Cochrane Library should be consulted for the most recent version of the review.

\section{Commentary}

The dental pulp refers to the contents in the centre of the tooth, which is made up nerves, blood vessels, lymphatics and cells essential for the normal development and functioning of the tooth. Irreversible pulpitis is a stage of pulpal inflammation often associated with irreversible pulpal damage, which would need endodontic intervention. The dynamics of this inflammatory process often lead to symptoms that may be acute or chronic in nature.

This Cochrane report looks at the effects of antibiotics in the management of irreversible pulpitis. The practice of administration of antibiotics to manage irreversible pulpitis and the misconception around their use by some dentists is highlighted by this review.

The primary disadvantage of this systematic review was that it was based on a single study with a small sample size. The authors acknowledge the absence of any reasonable long-term or short-term studies as the primary reason for the lack of evidence making it necessary for further studies. It should be appreciated however that studies of such a nature are often difficult to undertake, considering the distress that the participants are put through during the period of the study. Furthermore there is the question of ethics involved in conducting such a study.

The one included study by Nagle et al. ${ }^{1}$ did not indicate any significant benefits to patients administered with a dose of penicillin for the management of pain in relation to irreversible pulpitis. The role of bacteria in the pathogenesis of irreversible pulpitis caused by caries is well known, however, it has been demonstrated that the pulp is bacterium free in early irreversible pulpitis.

Reports of pain associated with irreversible pulpitis are variable with the classical symptom being one of lingering pain following the application of a thermal stimulus. The degree of pain experienced is often associated with the nature and extent of inflammation in the pulp.

However, factors such as pain threshold and physiological wellbeing of the patient are also important factors. The reported case in this review only indicates the patient's experience of pain (mild, moderate or severe), which is subjective and could be dependent on individual pain threshold.

Further, the study does not indicate at what point in the participants' pain experience they were required to take drugs for pain control (for example, when pain became unbearable or when they first perceived pain).

The lack of sufficient sample size in this reviewed study makes it difficult to identify the possible influence of variables. However, 
because irreversible pulpal pain is mainly associated with the inflammatory process and bacteria are rarely involved, the usefulness of antibiotics is questionable.

\section{Practice points}

- In the light of an increased potential for antibiotic resistance and insufficient evidence to indicate the benefits of antibiotics in controlling pain due to irreversible pulpitis, dentists should desist from the practice of prescribing antibiotics for early irreversible pulpitis.

- The use of analgesics in controlling pain due to irreversible pulpitis should be dependent on severity of perceived pain and in scenarios where there is a limitation in undertaking immediate pulp extirpation.
Overall the lack of sufficient studies seriously affects the objectives of this review and prevents the authors from making a reasonable recommendation on the usefulness of antibiotics for controlling pain associated with irreversible pulpitis.

Roy George

School of Dentistry and Oral Health, Griffith University, Queensland, Australia

1. Nagle D, Reader A, Beck M, Weaver J. Effect of systemic penicillin on pain in untreated irreversible pulpitis. Oral Surg Oral Med Oral Path Oral Radiol Endod 2000; 90: 636-640.

Evidence-Based Dentistry (2014), 15, 10-11. doi: 10.1038/sj.ebd.6400982 